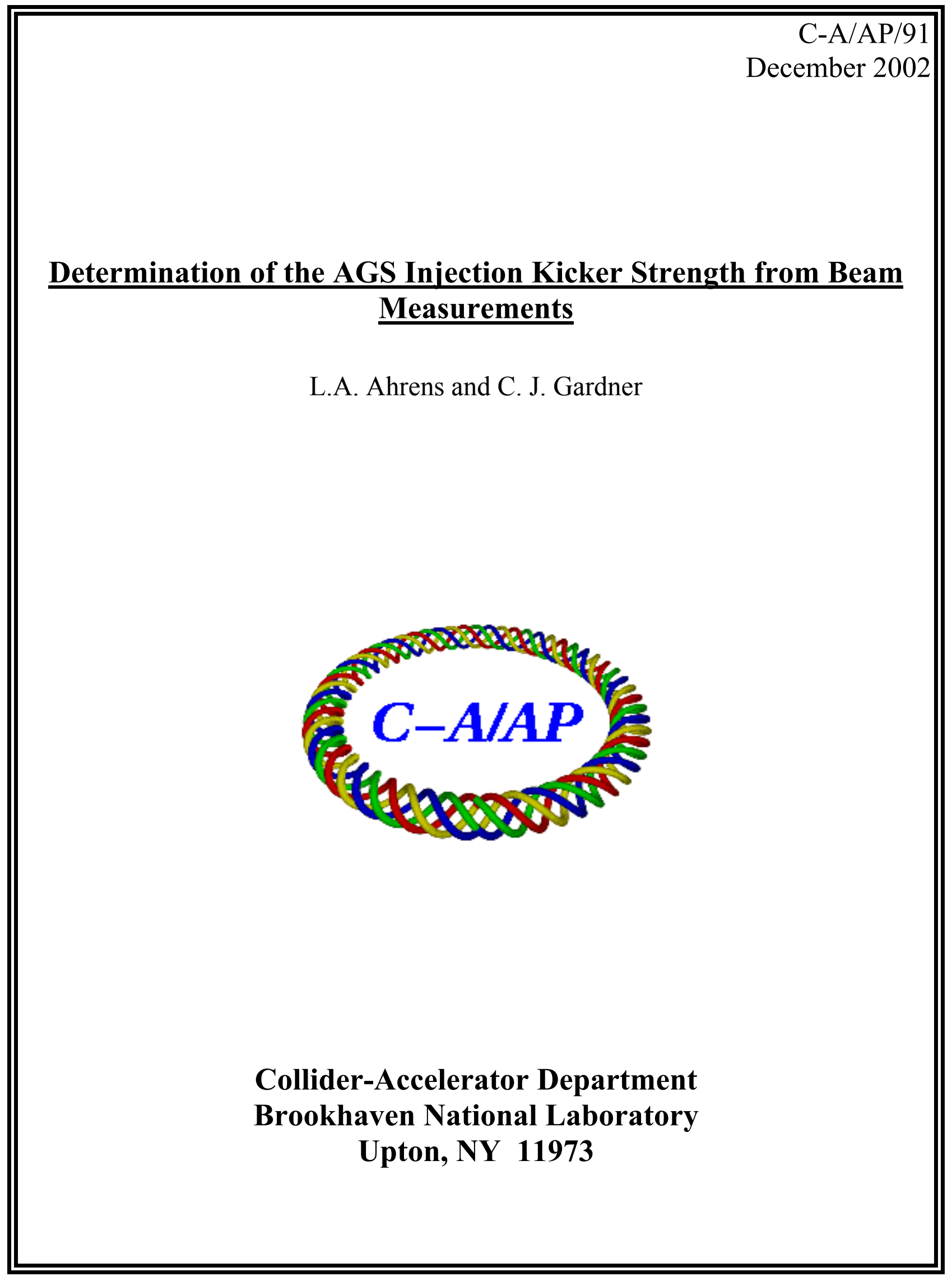




\title{
Determination of the AGS Injection Kicker Strength from Beam Measurements
}

\author{
L.A. Ahrens and C.J. Gardner
}

December 29, 2002

For several years now, during high-intensity proton operation, we have injected protons into AGS at a kinetic energy of $1.94 \mathrm{GeV}$, and have found that the AGS injection kicker does not have enough strength to kick the incoming beam onto the equilibrium orbit. This has led us to wonder how much the kicker is actually kicking the beam. A simple "back-of-the-envelope" calculation of the kicker's magnetic field gives a "reasonable" estimate of the strength, but no determination based upon measurements of the beam response to the kicker has ever been made. The FY2002 Polarized Proton and Proton SEB runs allowed for several opportunities to make the measurements.

The experimental method consisted of measuring the turn-by-turn positions of the injected beam at a given PUE (Pick-Up Electrode) for various settings of the kicker current. These data then can be used to obtain the angle (with respect to the equilibrium orbit) of the injected beam just downstream of the kicker. Fitting a line to a plot of the angle versus current then gives the kick angle per unit current. Since variation of the kicker current from its nominal setpoint interrupts the physics program and can produce enough loss at high intensity to inhibit the beam, the low intensity (some $1.7 \times 10^{11}$ protons per pulse at injection) polarized proton setup and the available study time during RHIC stores provided an excellent opportunity to acquire the turn-by-turn data. During the SEB running period, "pulse stealing" was used to acquire data at reduced intensity. In all, four sets of data were taken, two with the polarized proton setup and two with the proton SEB setup.

We report here on the data acquired, its analysis, and the resulting determination of the kicker strength. Theoretical calculations of the kicker strength are reviewed and compared with the experimental determination. 
The results of careful measurements of the kicker current also are given.

\section{Theoretical Calculation of Kicker Strength}

The AGS injection kicker is located in the A5 straight section and consists of three identical window frame ferrite magnets each 10 inches in length and spaced 2.5 inches apart. The mechanical aspects of the magnet design are documented in drawings D36-M-1185-2, D36-M-1186-2, D36-M-1187-2, D11-M-11976, D11-M-11977, D11-M-11985, D11-M-11986, and D11-M-11987-3. Excitation of the magnets is provided by a pulse forming network which is described in [1].

\section{1 "Back-of-the-Envelope" Calculation}

Figure 1 shows the magnet cross section. With current $I$ flowing out of the conductor on the right and into the conductor on the left, the magnetic field $B$ in the gap points down as indicated. Applying Ampère's law [2] one has

$$
\mu_{0} \oint \frac{\mathbf{B}}{\mu} \cdot d \mathbf{l}=\underbrace{\int_{G / 2}^{-G / 2} B d s}_{\text {Gap }}+\mu_{0} \underbrace{\int \frac{\mathbf{B}}{\mu} \cdot d \mathbf{l}}_{\text {Ferrite }}=\mu_{0} I
$$

where the line integral on the left is taken around the blue loop. Here $G=57.15 \mathrm{~mm}$ (2.25 inches) is the gap height, $\mu$ is the magnetic permeability in the ferrite, and $\mu_{0}=4 \pi \times 10^{-7} \mathrm{Tm} / \mathrm{A}$. The integral around the loop is the sum of the integral across the gap and the integral through the ferrite. We assume that $\mu$ is so much larger than $\mu_{0}$ that the integral through the ferrite can be neglected. We then have

$$
\int_{G / 2}^{-G / 2} B d s=\mu_{0} I
$$

We assume further that $B$ is independent of the position along the blue path in the gap. Then we have

$$
B G=\mu_{0} I
$$

and the field per unit current is

$$
B / I=\mu_{0} / G=2.1988 \times 10^{-5} \mathrm{~T} / \mathrm{A} .
$$


The effective length $l$ of each magnet is taken to be the length of the ferrite plus one gap length. This gives $l=311.15 \mathrm{~mm}$. The effective length of the entire kicker is then $L=3 l=933.45 \mathrm{~mm}$, and the integrated strength per unit current is

$$
B L / I=\mu_{0} L / G=2.0525 \times 10^{-5} \mathrm{Tm} / \mathrm{A} .
$$

\subsection{Opera Code Calculation}

An Opera Code calculation of the field has been carried out by Nick Tsoupas. This is a three-dimensional calculation which requires the details of the arrangement of ferrite and conductors in the magnets and at the magnet ends. These were obtained from the mechanical drawings and entered into the code. The calculation gives

$$
B / I=2.1741 \times 10^{-5} \mathrm{~T} / \mathrm{A}
$$

and

$$
B L / I=1.8718 \times 10^{-5} \mathrm{Tm} / \mathrm{A}
$$

where $B$ is the field in the center of the kicker, $B L$ is the integrated strength, and $I$ is the current. The effective length is then $L=861 \mathrm{~mm}$. Comparing with the results of the back-of-the-envelope calculation, we see that the value of $B / I$ obtained by the Opera Code is $1 \%$ lower and the value of $B L / I$ is $10 \%$ lower.

\subsection{Theoretical Kick Angle}

The angular kick delivered by the kicker is

$$
\phi=B L /(B \rho)
$$

and the kick per unit current is

$$
D=\phi / I=B L /(I B \rho)
$$

where $B \rho$ is the magnetic rigidity. Using the value of $B L / I$ obtained by the Opera Code, we obtain the values of $D$ listed in Table 1 for protons with various kinetic energies $K$, momenta $c p$, and rigidities $B \rho$.

Here the unit "mr/kA" is milliradians per kiloampère. The nominal kinetic energy at AGS injection for polarized proton operation is $1.5 \mathrm{GeV}$; for high-intensity proton SEB it is $1.94 \mathrm{GeV}$. 
Table 1: A5 Kick for Various Proton Kinetic Energies

\begin{tabular}{|c|c|c|c|}
\hline$K(\mathrm{GeV})$ & $c p(\mathrm{GeV})$ & $B \rho(\mathrm{Tm})$ & $D(\mathrm{mr} / \mathrm{kA})$ \\
\hline 1.50 & 2.25 & 7.5069 & 2.493 \\
1.60 & 2.36 & 7.8671 & 2.379 \\
1.70 & 2.47 & 8.2250 & 2.276 \\
1.80 & 2.57 & 8.5810 & 2.181 \\
1.90 & 2.68 & 8.9352 & 2.095 \\
1.94 & 2.72 & 9.0764 & 2.062 \\
2.00 & 2.78 & 9.2879 & 2.015 \\
\hline
\end{tabular}

\section{Kicker Current Measurement}

An accurate determination of the kicker strength per unit current requires an accurate measurement of the currents delivered to the three magnets of the kicker. These are measured with three current transformers, one for each magnet. The current transformers are located in the AGS L18A house and each gives an output of $1 \mathrm{~V}$ per $20 \mathrm{~A}$ of current when connected to an oscilliscope with $50 \Omega$ termination. A buffered sum of the outputs is available in the MCR at console 4 . This is called the "A5 Current" signal. The top half of Figure 2 shows the oscilliscope trace of this signal with $50 \Omega$ termination at the scope input. The bottom half shows the corresponding traces of the outputs from the three current transformers. These were obtained by D. Warburton in the L18A house with $50 \Omega$ termination at the scope inputs. Examination of the figure shows that the average amplitude of the current transformer traces is $57.9 \mathrm{~V}$ which gives a current of 1158 A. The corresponding amplitude of the "A5 Current" signal is $2.24 \mathrm{~V}$. Thus, if we let $V$ be the voltage of the "A5 Current" signal, then the kicker current in ampères is

$$
I=(1158 / 2.24) V(50 \Omega \text { termination }) .
$$

For the case in which the "A5 Current" signal is not terminated, we find

$$
I=(1139 / 4.20) V \text { (No termination) }
$$




\section{Turn-by-Turn Data and Analysis}

Data were taken on 16 and 21 January, 28 March, and 4 April, 2002. We shall refer to these as data sets 1, 2, 3 and 4 respectively. For each data set, a single bunch was injected onto the AGS injection porch and allowed to pass turn-by-turn through the PUE in the G14 straight section. This was done for several different settings of the kicker current. The turn-by-turn signals from the "inside" and "outside" plates of the PUE were captured on a digital ocsilliscope with the scope configured to take the sum and difference of the signals. The digitized "sum" and "differernce" signals were then analyzed by the PIP [3] program. Data sets 1 and 2 were taken during the polarized proton run with "high" gain amplifiers connected to the plates of the G14 PUE; sets 3 and 4 were taken during the proton SEB run with "low" gain amplifiers connected to the plates. The nominal kinetic energy of protons injected into AGS was 1.5 $\mathrm{GeV}$ for the polarized proton setup and $1.94 \mathrm{GeV}$ for the SEB setup.

\subsection{Sum and Difference Amplitudes}

Figure 3 shows a typical sum signal captured for the first 16 turns of the injected beam during the polarized proton run. Here the narrow peaks correspond to the turn-by-turn passage of a single bunch through the PUE. The narrowness of the peaks presented some difficulty for the original PIP algorithm which until now has dealt only with peaks whose width is approximately half the revolution period. The algorithm was modified so that it can cope with both narrow and broad peaks. Figure 4 shows a magnified view of the first two peaks in the sum signal and illustrates how the algorithm calculates the average amplitude of each peak. Here the points indicated by the red and blue circles are located by the algorithm and the area under each peak between the red and blue points is calculated. The baseline for this integration is taken to be the average of the signal at the red and blue points. The average amplitude of the peak is then the value of the integral divided by the integration interval. Figure $\mathbf{5}$ shows the corresponding red and blue circles for the first two peaks in the difference signal. These mark the points that occur at the same times as the circled points of the sum signal. The area under each peak is again calculated with the baseline for the integration taken to be the average of the signal at the red and blue points. The average amplitude of the peak is then again the value of the integral divided by the integration interval. 


\subsection{Turn-by-Turn Position at the PUE}

If we let "Sum" and "Diff" denote the turn-by-turn amplitudes obtained in this way, then the position of the beam on each passage through the PUE is just

$$
X=G\left(\frac{\text { Diff }}{\text { Sum }}\right)
$$

where $G$ is a constant that depends on the geometry of the PUE. Careful measurements by Ahrens [4] give $G=40 \mathrm{~mm}$. Figure 6 shows the turn-by-turn positions and fitted curve obtained by the Pip program for the sum and difference signals in Figures 3-5. Figures 7 and 8 show a typical difference signal and typical turn-by-turn positions obtained during the proton SEB run (with the "low" gain amplifiers connected to the G14 PUE).

\subsection{Initial Position and Angle at the Kicker}

Among the fitted parameters obtained by the PIP program are the tune $Q$, the amplitude $A$, and the phase $\phi$ of the turn-by-turn oscillations at the PUE. From the amplitude and phase one obtains the initial position and angle of the injected beam (with respect to the equilibrium orbit) at the PUE. These are

$$
X_{P}=A \cos \phi
$$

and

$$
X_{P}^{\prime}=-\frac{A}{\beta_{P}}\left\{\alpha_{P} \cos \phi+\sin \phi\right\}
$$

where $\alpha_{P}$ and $\beta_{P}$ are the Courant-Snyder parameters at the PUE. The initial position and angle, $X_{K}$ and $X_{K}^{\prime}$, of the beam just after the kicker are then given by

$$
\left(\begin{array}{c}
X_{K} \\
X_{K}^{\prime}
\end{array}\right)=\left(\begin{array}{rr}
M_{22} & -M_{12} \\
-M_{21} & M_{11}
\end{array}\right)\left(\begin{array}{l}
X_{P} \\
X_{P}^{\prime}
\end{array}\right)
$$

where

$$
\begin{gathered}
M_{11}=\sqrt{\beta_{P} / \beta_{K}}\left(\cos \psi+\alpha_{K} \sin \psi\right) \\
M_{22}=\sqrt{\beta_{K} / \beta_{P}}\left(\cos \psi-\alpha_{P} \sin \psi\right) \\
M_{12}=\sqrt{\beta_{K} \beta_{P}} \sin \psi
\end{gathered}
$$


and

$$
M_{21}=\frac{-1}{\sqrt{\beta_{K} \beta_{P}}}\left\{\left(\alpha_{P}-\alpha_{K}\right) \cos \psi+\left(1+\alpha_{K} \alpha_{P}\right) \sin \psi\right\} .
$$

Here $\alpha_{K}$ and $\beta_{K}$ are the Courant-Snyder parameters at the Kicker, and $\psi$ is the betatron phase advance from kicker to PUE. (The kicker is taken to be a thin dipole located at the center of the A5 straight.) The

Courant-Snyder parameters are obtained from the MAD model of the AGS lattice and the phase advance is given by scaling the model phase advance according to the measured tune $Q$.

Using (13), (14), and (16-19) in (15) we find

$$
X_{K}=A \sqrt{\beta_{K} / \beta_{P}} \cos (\psi-\phi)
$$

and

$$
X_{K}^{\prime}=\frac{A}{\sqrt{\beta_{K} \beta_{P}}}\left\{\sin (\psi-\phi)-\alpha_{K} \cos (\psi-\phi)\right\}
$$

which give $X_{K}$ and $X_{K}^{\prime}$ in terms of the fitted parameters $A$ and $\phi$, the Courant-Snyder parameters $\alpha_{K}, \beta_{K}$, and $\beta_{P}$, and the betatron phase advance $\psi$.

\subsection{Courant-Snyder Invariant}

The Courant-Snyder invariant is

$$
W=\gamma_{P} X_{P}^{2}+2 \alpha_{P} X_{P} X_{P}^{\prime}+\beta_{P} X_{P}^{\prime 2}
$$

where

$$
\gamma_{P}=\left(1+\alpha_{P}^{2}\right) / \beta_{P}
$$

We then have

$$
\beta_{P} W=X_{P}^{2}+\left(\alpha_{P} X_{P}+\beta_{P} X_{P}^{\prime}\right)^{2}
$$

and using (13) and (14) we find

$$
\beta_{P} W=A^{2} .
$$

Thus

$$
W=A^{2} / \beta_{P}
$$

which gives $W$ in terms of the fitted parameter $A$ and the Courant-Snyder parameter $\beta_{P}$. Since $W$ is invariant, we also have

$$
W=\gamma_{K} X_{K}^{2}+2 \alpha_{K} X_{K} X_{K}^{\prime}+\beta_{K} X_{K}^{\prime 2}
$$


where

$$
\gamma_{K}=\left(1+\alpha_{K}^{2}\right) / \beta_{K}
$$

Thus

$$
\beta_{K} W=X_{K}^{2}+\left(\alpha_{K} X_{K}+\beta_{K} X_{K}^{\prime}\right)^{2}
$$

and therefore

$$
\left(\alpha_{K} X_{K}+\beta_{K} X_{K}^{\prime}\right)^{2}=A^{2} \beta_{K} / \beta_{P}-X_{K}^{2} .
$$

For the special case in which $X_{K}=0$, this reduces to

$$
X_{K}^{\prime 2}=\frac{A^{2}}{\beta_{K} \beta_{P}}
$$

and we see that the magnitude of $X_{K}^{\prime}$ is then completely determined by $A$, $\beta_{K}$ and $\beta_{P}$.

\section{Determination of Kicker Strength}

The parameters $A, W, X_{K}$ and $X_{K}^{\prime}$ obtained from the PIP analysis of data sets 1, 2, 3 and 4 are listed in Tables 3, 4, 5, and $\mathbf{6}$ respectively. Here $W$, $X_{K}$, and $X_{K}^{\prime}$ have been calculated according to equations (26), (20), and (21). The kicker currents $I$ have been obtained from (10) and (11) using the measured amplitudes $V$ of the "A5 Current" signal. Note that in each table, $W$ is very small for some settings of the kicker current. Equation (29) then implies that $X_{K}$ also must be very small. Since $X_{K}$ does not change as the kicker current is varied, it then must be small for all entries in the tables (and indeed it is). (We are assuming here that position and angle of the incoming beam upstream of the kicker do not change.) Thus, to a good approximation $X_{K}=0$ and therefore, according to equation (31),

$$
X_{K}^{\prime}=\frac{ \pm A}{\sqrt{\beta_{P} \beta_{K}}} .
$$

This result can be used to check the values of $X_{K}^{\prime}$ obtained using equation (21). Using the values $\beta_{K}=22.066$ and $\beta_{P}=15.768$ obtained from the MAD model of the AGS lattice we obtain the values of $A / \sqrt{\beta_{P} \beta_{K}}$ listed in the last column of the tables. These are in good agreement with the listed values of $X_{K}^{\prime}$. This shows that the phase advance $\psi$ used in (20) and (21) is correct, and in fact must be such that $\sin ^{2}(\psi-\phi) \approx 1$. 
Figures 9, 10, 11, and $\mathbf{1 2}$ are plots of the angles $X_{K}^{\prime}$ versus current $I$ obtained from Tables 3, 4, 5, and 6 respectively. Fitting the expression

$$
X_{K}^{\prime}(I)=C+D I
$$

to the data we obtain the red and blue lines shown in the figures. The red lines are the result of fitting to all of the data points; the blue lines are the result of fitting only to points with currents greater than $900 \mathrm{~A}$. The slope $D$ of each line is the angular kick delivered by the kicker per unit current. The intercept $C$ is the angle $X_{K}^{\prime}$ at zero current. The fitted values of $C$ and $D$ are listed in Table 2 along with the theoretical values of $D$ obtained from the Opera Code calculation. The units of $D$ are mrad/kA; those of $C$ are mrad. Comparing numbers, we see that the slopes of the blue lines agree well with the theoretical values of $D$. The slopes of the red lines are substantially lower. We believe this is due to beam getting scraped off on the machine apertures for the lower settings of the kicker current.

Table 2: Fitted and Theoretical Parameters

\begin{tabular}{|c|c|c|c|c|c|c|}
\hline Data & $K(\mathrm{GeV})$ & Plot & Line & $D$ & Theory & $C$ \\
\hline Set 1 & 1.5 & Figure 9 & red & $1.62(14)$ & 2.493 & $-1.86(14)$ \\
$($ Table 3) & & & & & & \\
\hline Set 2 & 1.5 & Figure 10 & red & $1.80(12)$ & 2.493 & $-2.08(12)$ \\
(Table 4) & & & blue & $2.21(25)$ & 2.493 & $-2.52(26)$ \\
\hline Set 3 & 1.94 & \multirow{2}{*}{ Figure 11 } & red & $1.49(10)$ & 2.062 & $-1.71(10)$ \\
(Table 5) & & & blue & $1.91(22)$ & 2.062 & $-2.17(23)$ \\
\hline Set 4 & 1.94 & \multirow{2}{*}{ Figure 12 } & red & $1.50(04)$ & 2.062 & $-1.72(03)$ \\
(Table 6) & & & blue & $2.22(20)$ & 2.062 & $-2.45(21)$ \\
\hline
\end{tabular}

Given the good agreement between blue line slopes and the theoretical values of $D$, we take the kicker integrated strength per unit current to be that given by the Opera Code calculation,

$$
B L / I=1.8718 \times 10^{-5} \mathrm{Tm} / \mathrm{A} .
$$




\section{References}

[1] W. Zhang, R. Sanders, A. Soukas, J. Tuozzolo, "An Overview of the Fast Injection-Extraction Kicker Systems of the Brookhaven AGS-Booster Complex", Proceedings of the 1999 Particle Accelerator Conference, New York, 1999, pp. 1264-1266.

[2] J.D. Jackson, "Classical Electrodynamics", Third Edition, John Wiley \& Sons, Inc., New York, 1998, Chapter 5

[3] C.J. Gardner, L. Ahrens, N. Williams, "Turn-by-Turn Analysis of Proton and Gold Beams at Injection in the AGS Booster", Proceedings of the 1999 Particle Accelerator Conf., PAC-99, New York, N.Y., USA, 29 March-2 April 1999.

[4] L. A. Ahrens, "AGS PUE Geometry Factor and Normalization", AGS/AD/Tech Note 459, March 5, 1997. 


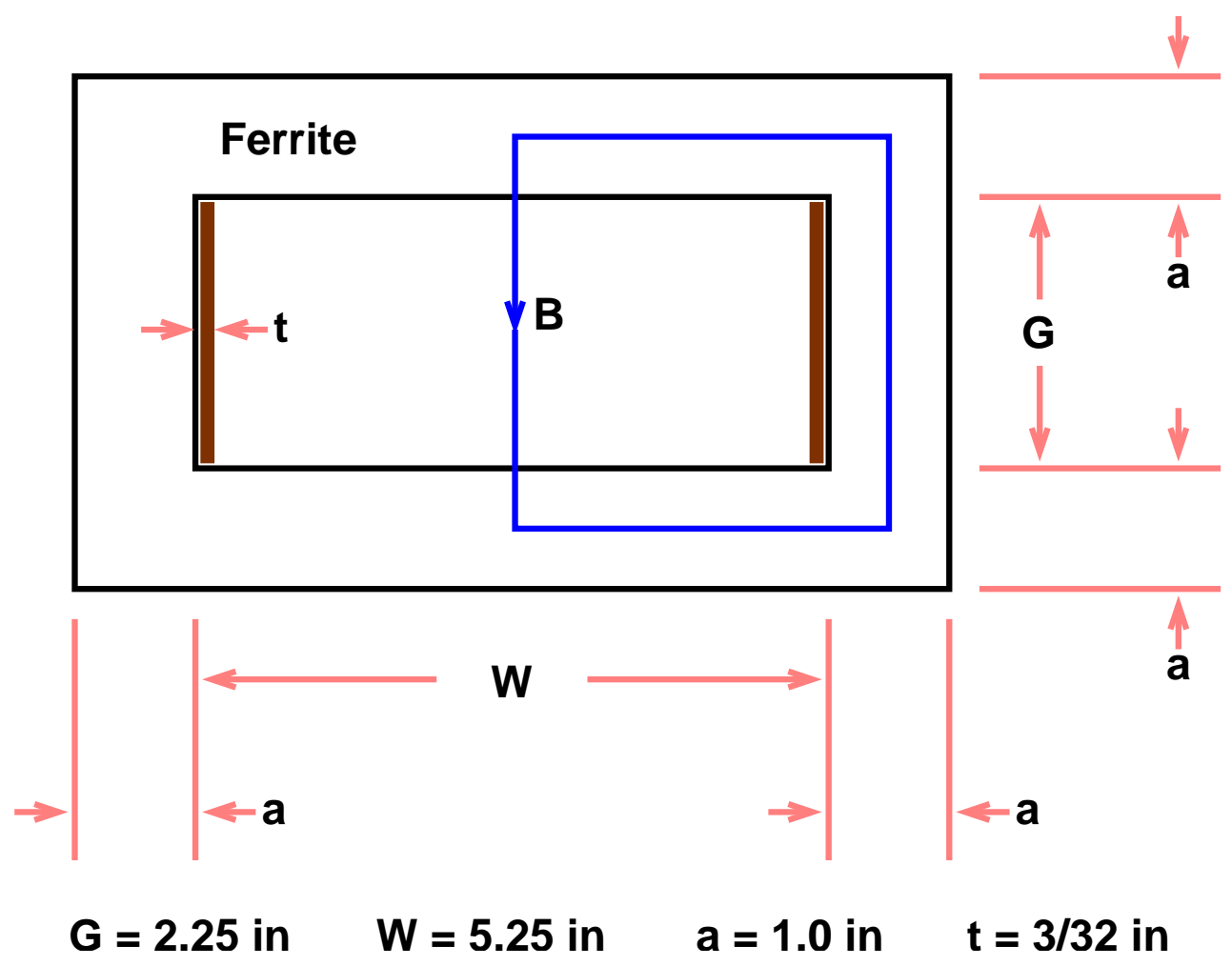

Figure 1: A5 Kicker Cross Section. The indicated dimensions have been obtained from drawings D36-M-1185-2, D36-M-1186-2, D36-M-11872, D11-M-11976, D11-M-11977, D11-M-11985, D11-M-11986, and D11-M-11987-3. The brown rectangles indicate copper conductor. 


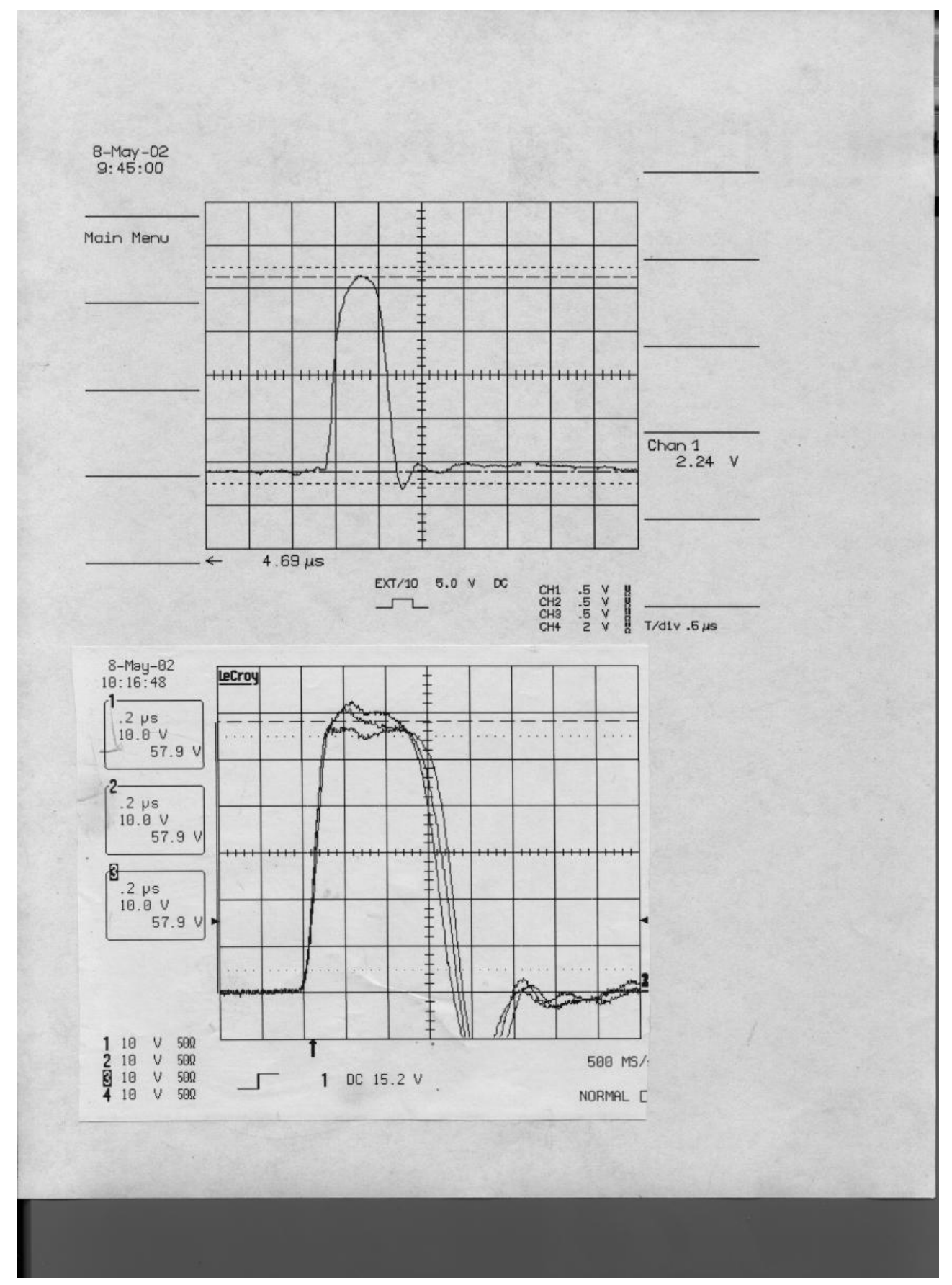

Figure 2: A5 Kicker Waveforms 


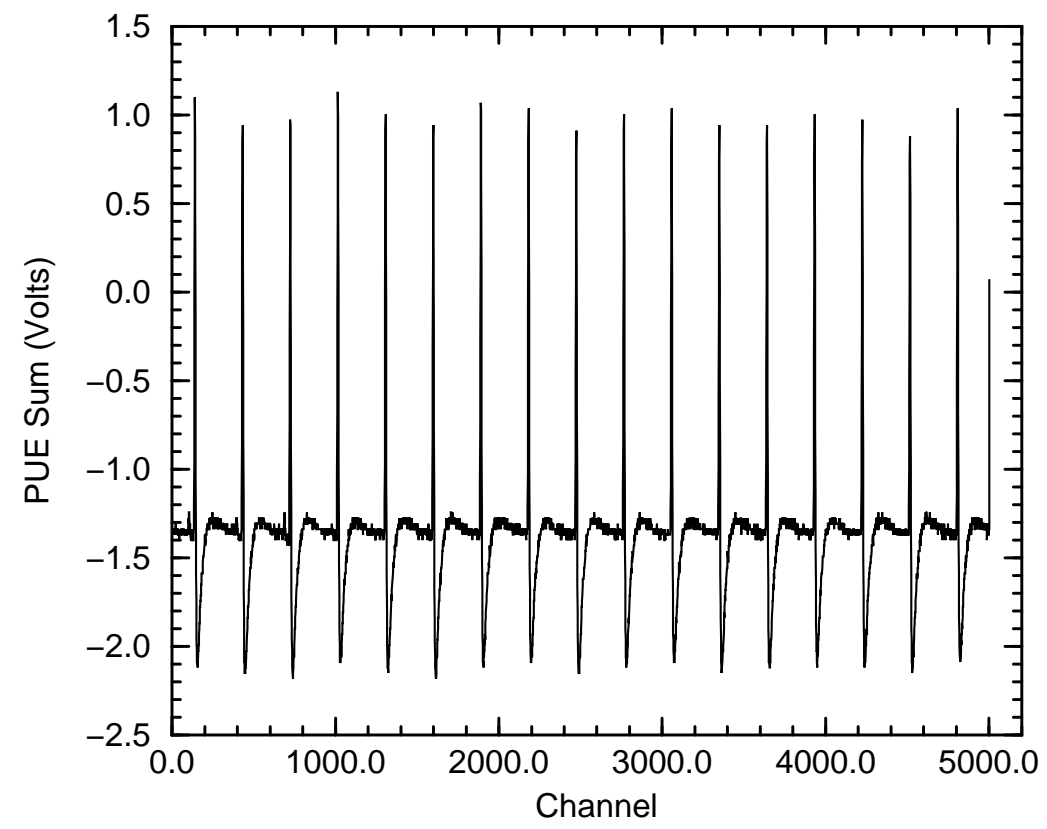

Figure 3: G14 Horizontal Sum Signal

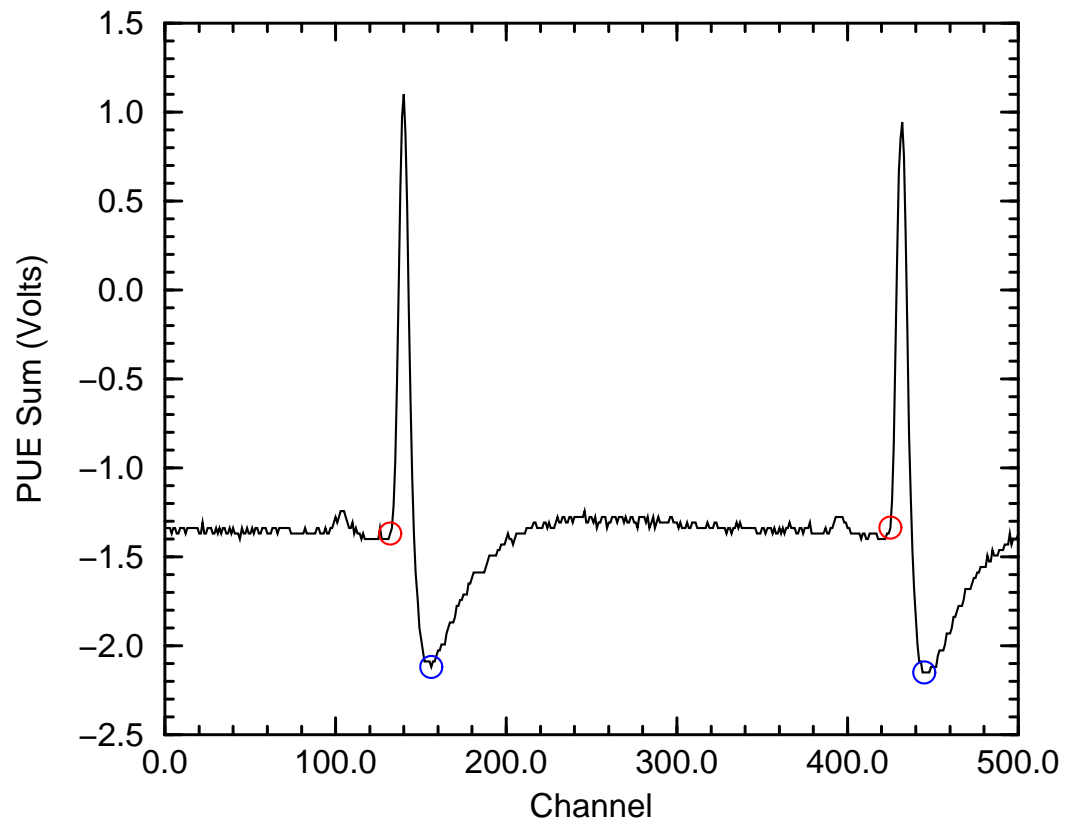

Figure 4: Magnified Sum Signal 


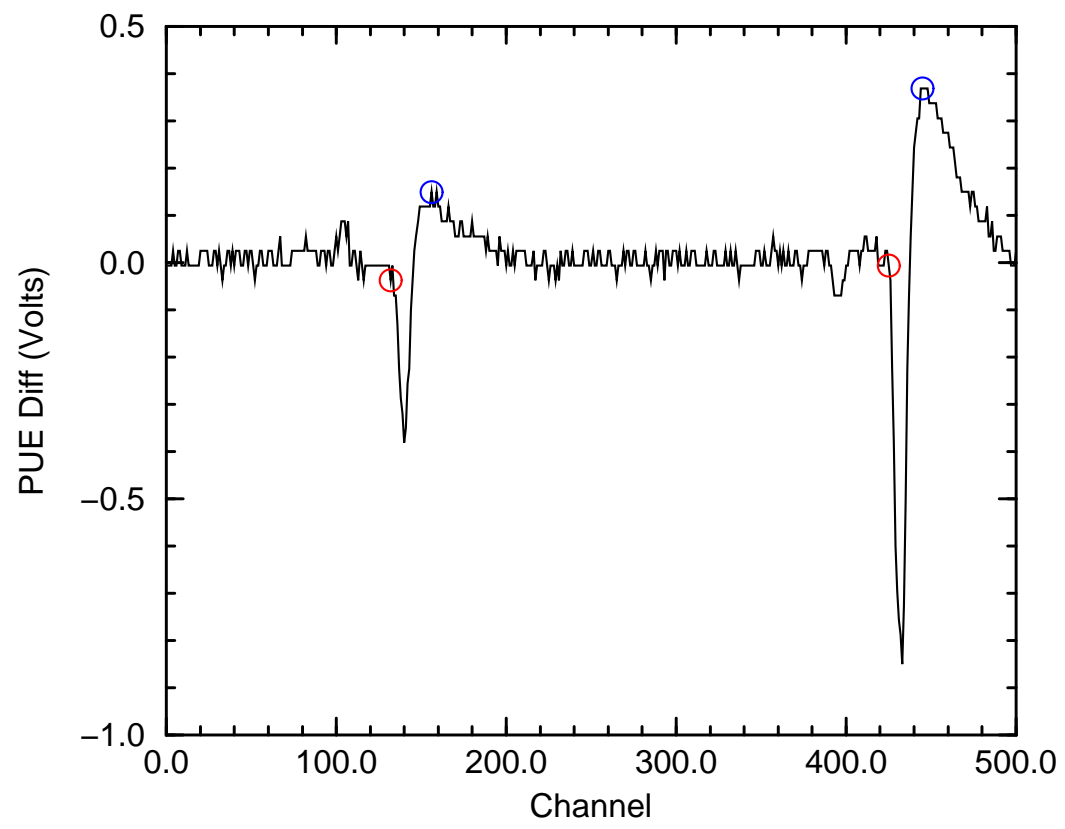

Figure 5: Magnified Diff Signal

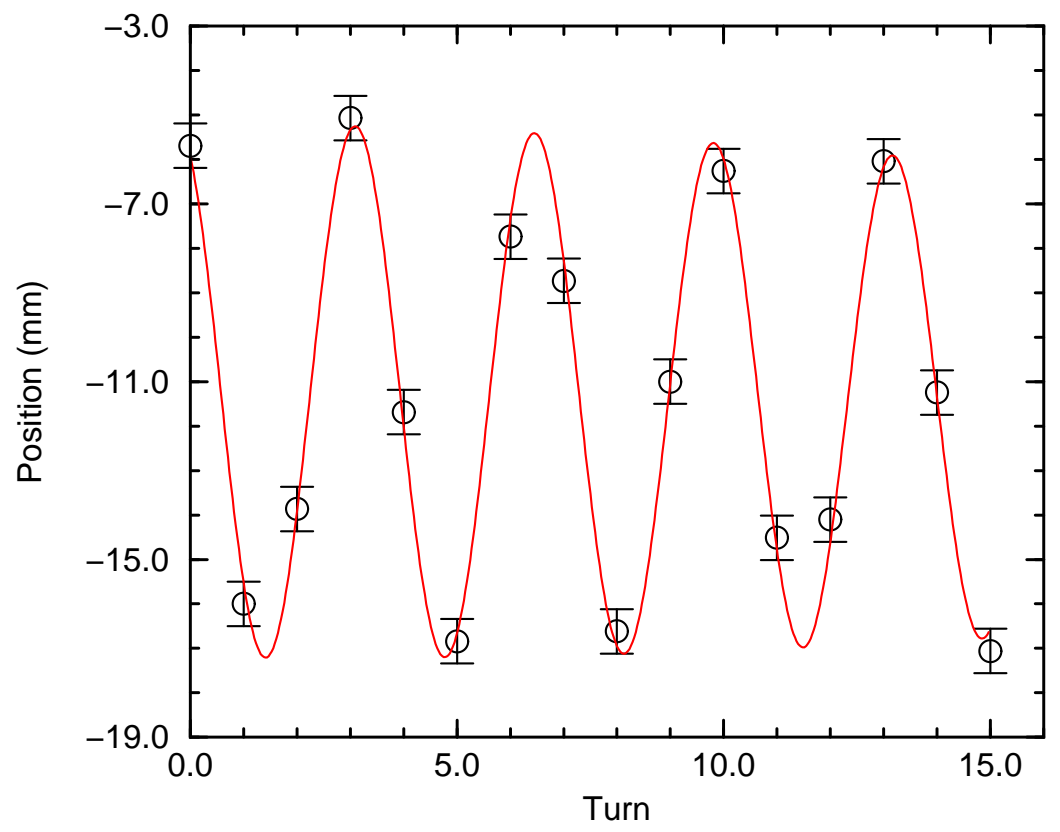

Figure 6: Position-vs-Turn at G14 PUE and Fitted Curve 


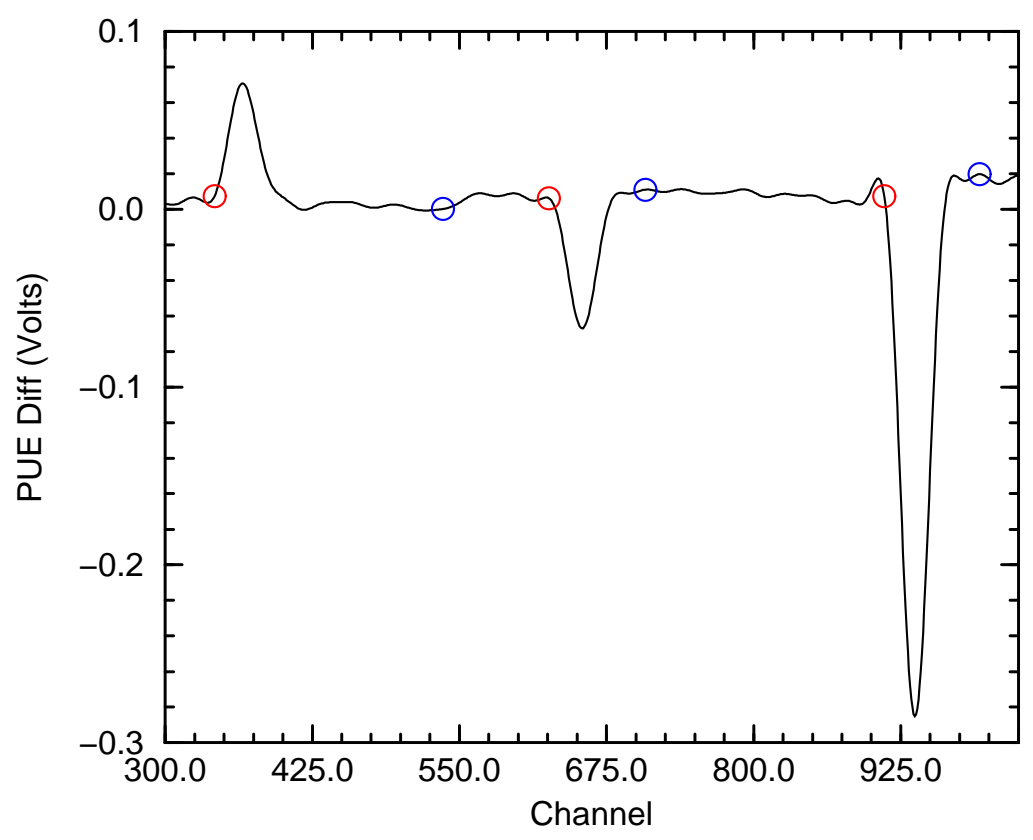

Figure 7: Magnified Diff Signal

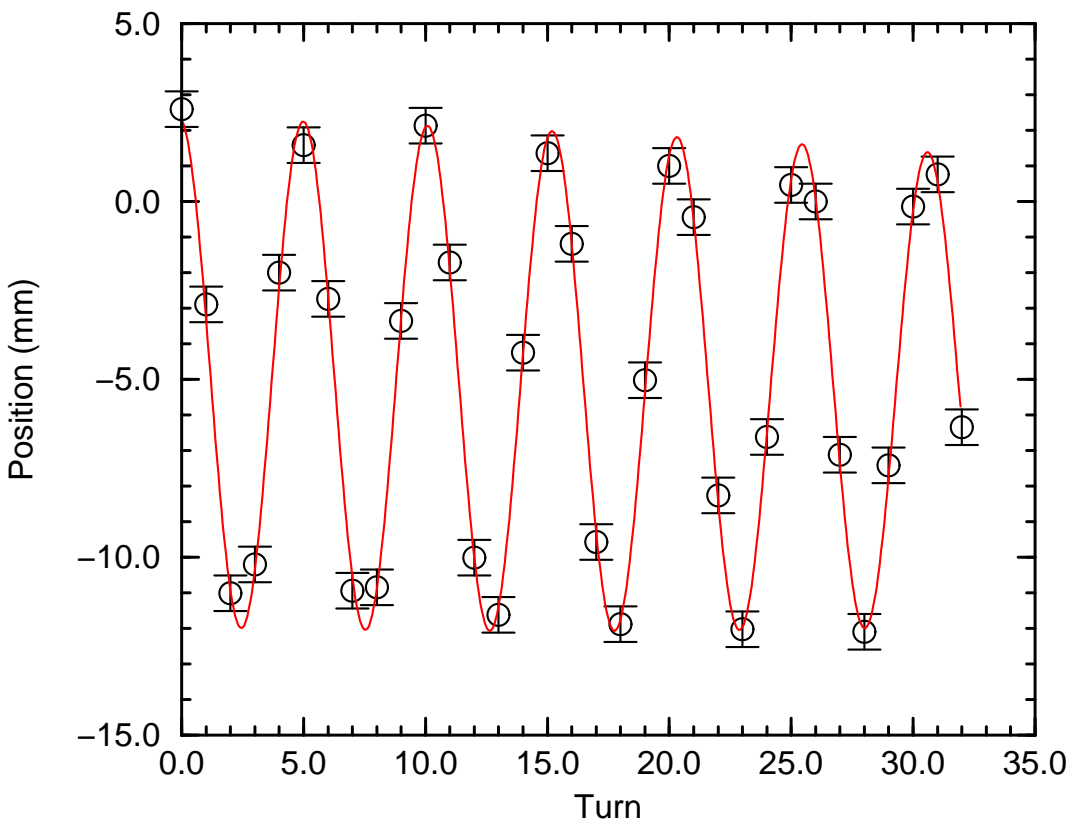

Figure 8: Position-vs-Turn at G14 PUE and Fitted Curve 
Table 3: PIP Data Set 1 (16 January 2002)

\begin{tabular}{|c|c|c|c|r|r|r|c|}
\hline File & $V$ & $I$ & $A(\mathrm{~mm})$ & $W$ & $X_{K}(\mathrm{~mm})$ & $X_{K}^{\prime}(\mathrm{mrad})$ & $A / \sqrt{\beta_{P} \beta_{K}}$ \\
\hline $\mathrm{a}$ & 4.20 & 1139 & $1.62(28)$ & $0.17(6)$ & $1.4(1.3)$ & $-0.061(46)$ & $0.087(15)$ \\
$\mathrm{f}$ & 4.20 & 1139 & $0.88(29)$ & $0.05(3)$ & $1.0(1.2)$ & $0.009(38)$ & $0.047(16)$ \\
$\mathrm{g}$ & 4.20 & 1139 & $0.85(27)$ & $0.05(3)$ & $-0.8(0.9)$ & $0.029(56)$ & $0.046(14)$ \\
$\mathrm{h}$ & 4.20 & 1139 & $0.66(28)$ & $0.03(2)$ & $0.8(1.0)$ & $0.007(40)$ & $0.035(15)$ \\
\hline $\mathrm{d}$ & 3.57 & 968 & $5.46(25)$ & $1.89(18)$ & $-0.6(1.2)$ & $-0.292(43)$ & $0.293(13)$ \\
$\mathrm{e}$ & 3.57 & 968 & $6.01(26)$ & $2.29(20)$ & $-0.1(1.2)$ & $-0.322(46)$ & $0.322(14)$ \\
$\mathrm{i}$ & 3.57 & 968 & $5.61(26)$ & $1.99(19)$ & $-1.0(1.2)$ & $-0.297(43)$ & $0.301(14)$ \\
$\mathrm{k}$ & 3.57 & 968 & $4.97(26)$ & $1.56(16)$ & $1.0(1.3)$ & $-0.262(47)$ & $0.266(14)$ \\
\hline $\mathrm{b}$ & 3.04 & 824 & $9.67(26)$ & $5.93(32)$ & $0.0(1.2)$ & $-0.518(47)$ & $0.518(14)$ \\
\hline $\mathrm{c}$ & 2.67 & 724 & $12.52(26)$ & $9.94(41)$ & $-0.4(1.2)$ & $-0.671(46)$ & $0.671(14)$ \\
\hline
\end{tabular}

Table 4: PIP Data Set 2 (21 January 2002)

\begin{tabular}{|c|c|c|c|c|r|r|c|}
\hline File & $V$ & $I$ & $A(\mathrm{~mm})$ & $W$ & $X_{K}(\mathrm{~mm})$ & $X_{K}^{\prime}(\mathrm{mrad})$ & $A / \sqrt{\beta_{P} \beta_{K}}$ \\
\hline $\mathrm{a}$ & 4.18 & 1134 & $0.34(26)$ & $0.01(1)$ & $0.2(0.9)$ & $0.016(26)$ & $0.018(14)$ \\
$\mathrm{b}$ & 4.18 & 1134 & $1.03(49)$ & $0.07(6)$ & $1.2(0.8)$ & $0.010(74)$ & $0.055(26)$ \\
$\mathrm{c}$ & 4.18 & 1134 & $0.84(29)$ & $0.04(3)$ & $-0.9(1.5)$ & $0.014(29)$ & $0.045(16)$ \\
\hline $\mathrm{d}$ & 3.99 & 1082 & $2.25(27)$ & $0.32(08)$ & $1.4(1.3)$ & $-0.104(50)$ & $0.121(14)$ \\
$\mathrm{e}$ & 3.99 & 1082 & $2.84(27)$ & $0.51(10)$ & $1.0(1.3)$ & $-0.145(50)$ & $0.152(14)$ \\
$\mathrm{f}$ & 3.99 & 1082 & $4.46(27)$ & $1.26(15)$ & $0.8(1.3)$ & $-0.236(52)$ & $0.239(14)$ \\
\hline $\mathrm{g}$ & 3.80 & 1031 & $4.66(26)$ & $1.38(15)$ & $0.8(1.2)$ & $-0.247(50)$ & $0.250(14)$ \\
$\mathrm{h}$ & 3.80 & 1031 & $4.47(26)$ & $1.26(15)$ & $0.3(1.2)$ & $-0.239(48)$ & $0.240(14)$ \\
\hline $\mathrm{i}$ & 3.64 & 987 & $5.32(26)$ & $1.80(17)$ & $-0.1(1.2)$ & $-0.285(46)$ & $0.285(14)$ \\
$\mathrm{j}$ & 3.64 & 987 & $6.47(28)$ & $2.65(23)$ & $1.3(1.3)$ & $-0.342(50)$ & $0.347(15)$ \\
\hline $\mathrm{k}$ & 3.44 & 933 & $8.54(26)$ & $4.63(28)$ & $-0.9(1.2)$ & $-0.456(47)$ & $0.458(14)$ \\
$\mathrm{l}$ & 3.44 & 933 & $8.45(27)$ & $4.53(29)$ & $0.4(1.3)$ & $-0.453(49)$ & $0.453(14)$ \\
\hline $\mathrm{m}$ & 3.17 & 860 & $9.90(26)$ & $6.22(32)$ & $0.8(1.2)$ & $-0.530(48)$ & $0.531(14)$ \\
$\mathrm{n}$ & 3.17 & 860 & $10.06(26)$ & $6.42(33)$ & $-0.4(1.2)$ & $-0.539(46)$ & $0.539(14)$ \\
\hline $\mathrm{o}$ & 3.01 & 816 & $10.99(26)$ & $7.66(37)$ & $-1.0(1.2)$ & $-0.587(46)$ & $0.589(14)$ \\
$\mathrm{p}$ & 3.01 & 816 & $10.83(26)$ & $7.43(36)$ & $-1.8(1.2)$ & $-0.575(44)$ & $0.581(14)$ \\
\hline $\mathrm{q}$ & 2.87 & 778 & $12.44(26)$ & $9.81(42)$ & $0.0(1.2)$ & $-0.667(47)$ & $0.667(14)$ \\
$\mathrm{r}$ & 2.87 & 778 & $12.07(26)$ & $9.23(40)$ & $-1.0(1.2)$ & $-0.645(45)$ & $0.647(14)$ \\
\hline
\end{tabular}




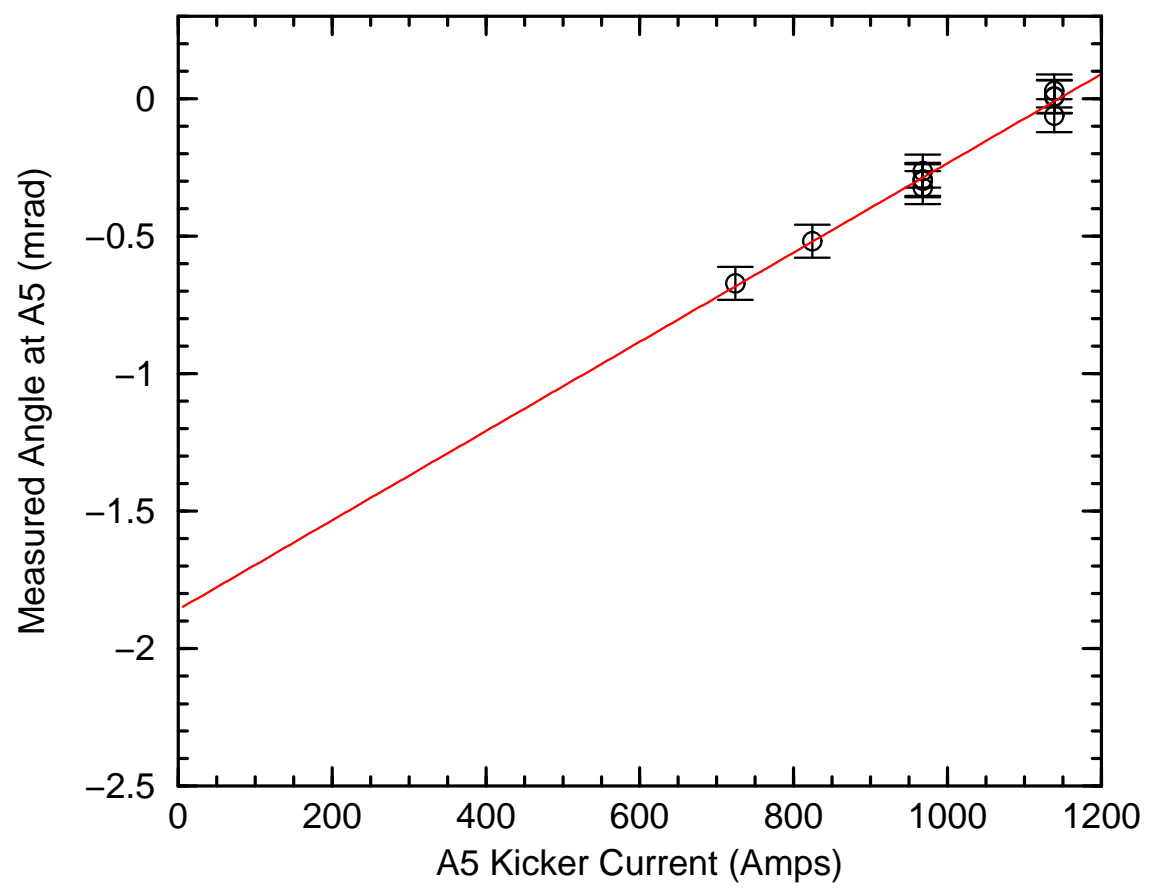

Figure 9: Data Set 1 with Fitted Line

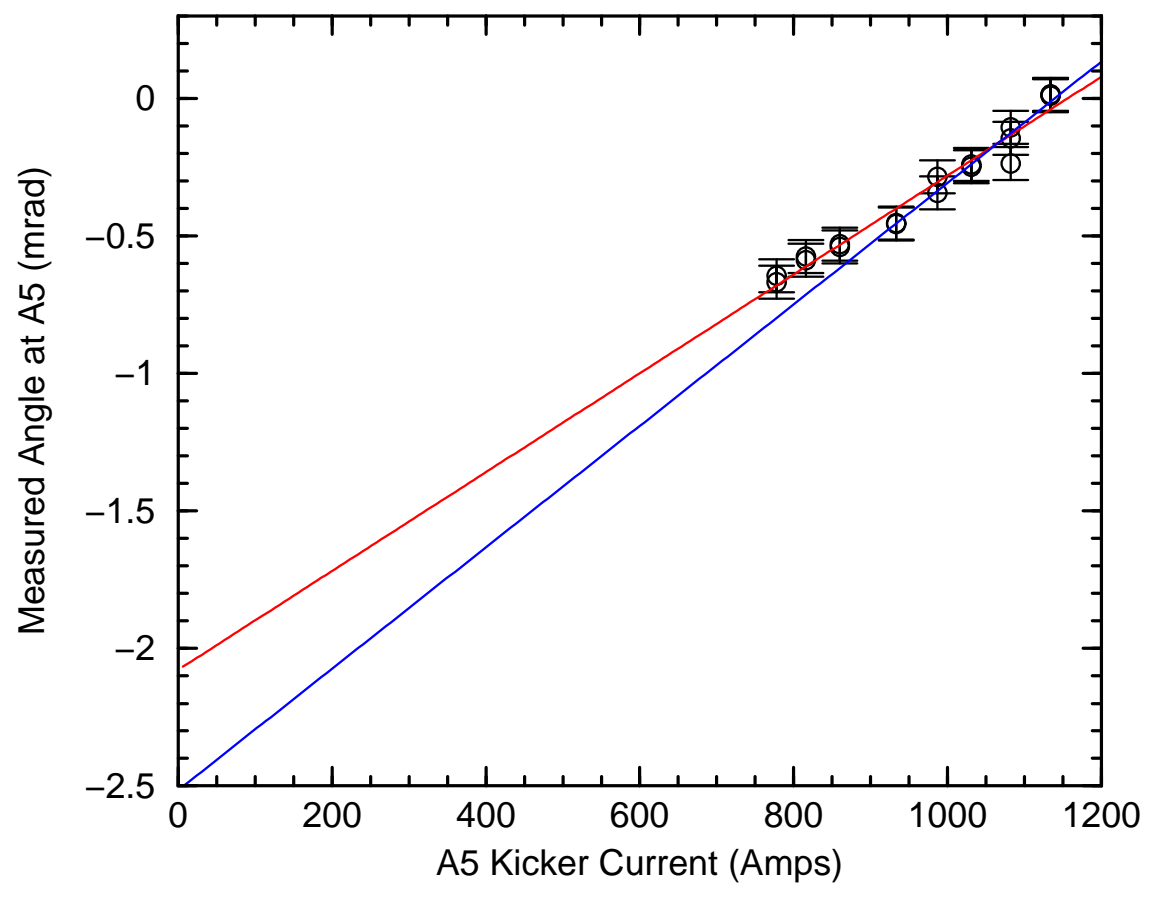

Figure 10: Data Set 2 with Fitted Lines 
Table 5: PIP Data Set 3 (28 March 2002)

\begin{tabular}{|c|c|c|c|c|c|r|c|}
\hline File & $V$ & $I$ & $A(\mathrm{~mm})$ & $W$ & $X_{K}(\mathrm{~mm})$ & $X_{K}^{\prime}(\mathrm{mrad})$ & $A / \sqrt{\beta_{P} \beta_{K}}$ \\
\hline $\mathrm{A}$ & 2.21 & 1142 & $2.36(19)$ & $0.35(6)$ & $-2.8(1.0)$ & $-0.005(24)$ & $0.127(10)$ \\
$\mathrm{B}$ & 2.21 & 1142 & $1.92(19)$ & $0.24(5)$ & $-2.2(1.0)$ & $0.034(25)$ & $0.103(10)$ \\
\hline $\mathrm{C}$ & 2.05 & 1060 & $2.95(18)$ & $0.55(7)$ & $-1.5(0.6)$ & $-0.143(15)$ & $0.158(10)$ \\
$\mathrm{D}$ & 2.05 & 1060 & $2.95(18)$ & $0.55(7)$ & $-0.9(0.7)$ & $-0.153(14)$ & $0.158(10)$ \\
\hline $\mathrm{E}$ & 1.86 & 962 & $7.19(18)$ & $3.28(17)$ & $-5.0(0.7)$ & $-0.311(17)$ & $0.386(10)$ \\
$\mathrm{F}$ & 1.86 & 962 & $7.54(19)$ & $3.61(18)$ & $-4.5(0.7)$ & $-0.349(16)$ & $0.404(10)$ \\
\hline $\mathrm{G}$ & 1.65 & 853 & $8.84(19)$ & $4.96(21)$ & $-2.1(0.7)$ & $-0.464(14)$ & $0.474(10)$ \\
$\mathrm{H}$ & 1.65 & 853 & $8.51(19)$ & $4.59(20)$ & $-2.0(0.7)$ & $-0.447(14)$ & $0.456(10)$ \\
\hline $\mathrm{I}$ & 1.54 & 796 & $9.36(19)$ & $5.57(22)$ & $-1.2(0.8)$ & $-0.499(14)$ & $0.502(10)$ \\
$\mathrm{J}$ & 1.54 & 796 & $9.28(19)$ & $5.46(22)$ & $-1.4(0.8)$ & $-0.493(14)$ & $0.498(10)$ \\
\hline
\end{tabular}

Table 6: PIP Data Set 4 (4 April 2002)

\begin{tabular}{|c|c|c|c|c|r|r|l|}
\hline File & $V$ & $I$ & $A(\mathrm{~mm})$ & $W(\mathrm{~mm} \mathrm{mr})$ & $X_{K}(\mathrm{~mm})$ & $X_{K}^{\prime}(\mathrm{mrad})$ & $A / \sqrt{\beta_{P} \beta_{K}}$ \\
\hline $\mathrm{a}$ & 2.17 & 1122 & $0.36(26)$ & $0.008(12)$ & $-0.3(1.4)$ & $0.014(21)$ & $0.019(14)$ \\
\hline $\mathrm{b}$ & 0.96 & 496 & $17.05(19)$ & $18.44(42)$ & $2.1(0.9)$ & $-0.909(16)$ & $0.914(10)$ \\
$\mathrm{c}$ & 0.81 & 419 & $18.70(20)$ & $22.17(47)$ & $1.4(0.9)$ & $-1.000(16)$ & $1.003(11)$ \\
$\mathrm{d}$ & 0.90 & 465 & $17.51(19)$ & $19.46(43)$ & $2.8(0.9)$ & $-0.931(16)$ & $0.939(10)$ \\
$\mathrm{e}$ & 1.05 & 543 & $16.62(19)$ & $17.53(40)$ & $0.8(0.8)$ & $-0.890(16)$ & $0.891(10)$ \\
\hline $\mathrm{f}$ & 1.17 & 605 & $15.64(19)$ & $15.52(37)$ & $1.1(0.8)$ & $-0.837(16)$ & $0.838(10)$ \\
$\mathrm{g}$ & 1.17 & 605 & $16.14(19)$ & $16.53(39)$ & $0.5(0.8)$ & $-0.865(15)$ & $0.865(10)$ \\
\hline $\mathrm{h}$ & 1.28 & 662 & $14.20(19)$ & $12.80(34)$ & $0.4(0.8)$ & $-0.761(15)$ & $0.761(10)$ \\
$\mathrm{i}$ & 1.28 & 662 & $13.73(19)$ & $11.95(33)$ & $4.4(0.9)$ & $-0.709(18)$ & $0.736(10)$ \\
\hline $\mathrm{j}$ & 1.38 & 713 & $12.60(19)$ & $10.07(30)$ & $2.0(0.8)$ & $-0.669(16)$ & $0.675(10)$ \\
$\mathrm{k}$ & 1.38 & 713 & $12.12(19)$ & $9.31(29)$ & $1.1(0.8)$ & $-0.648(16)$ & $0.650(10)$ \\
\hline $\mathrm{l}$ & 1.55 & 801 & $10.67(19)$ & $7.22(25)$ & $1.0(0.8)$ & $-0.570(16)$ & $0.572(10)$ \\
$\mathrm{m}$ & 1.55 & 801 & $10.73(19)$ & $7.30(26)$ & $0.9(0.8)$ & $-0.574(16)$ & $0.575(10)$ \\
\hline $\mathrm{n}$ & 1.65 & 853 & $9.32(19)$ & $5.51(22)$ & $-0.4(0.8)$ & $-0.499(15)$ & $0.500(10)$ \\
$\mathrm{o}$ & 1.65 & 853 & $9.61(19)$ & $5.86(23)$ & $-0.4(0.8)$ & $-0.515(15)$ & $0.515(10)$ \\
\hline $\mathrm{p}$ & 1.80 & 931 & $7.14(18)$ & $3.24(17)$ & $-0.5(0.8)$ & $-0.382(15)$ & $0.383(10)$ \\
$\mathrm{q}$ & 1.80 & 931 & $7.21(18)$ & $3.29(17)$ & $-0.8(0.8)$ & $-0.385(14)$ & $0.387(10)$ \\
\hline $\mathrm{r}$ & 1.97 & 1018 & $3.66(18)$ & $0.85(09)$ & $-1.0(0.7)$ & $-0.191(14)$ & $0.196(10)$ \\
$\mathrm{s}$ & 1.97 & 1018 & $3.38(18)$ & $0.73(08)$ & $0.0(0.8)$ & $-0.181(15)$ & $0.181(10)$ \\
\hline $\mathrm{t}$ & 2.05 & 1060 & $1.53(18)$ & $0.15(04)$ & $0.3(0.9)$ & $-0.081(17)$ & $0.082(10)$ \\
$\mathrm{u}$ & 2.05 & 1060 & $1.31(19)$ & $0.11(03)$ & $1.0(1.1)$ & $-0.048(23)$ & $0.070(10)$ \\
\hline $\mathrm{v}$ & 2.14 & 1106 & $0.74(19)$ & $0.04(02)$ & $0.8(0.9)$ & $0.016(23)$ & $0.040(10)$ \\
$\mathrm{w}$ & 2.14 & 1106 & $0.60(21)$ & $0.02(02)$ & $0.7(0.9)$ & $0.011(26)$ & $0.032(11)$ \\
\hline
\end{tabular}




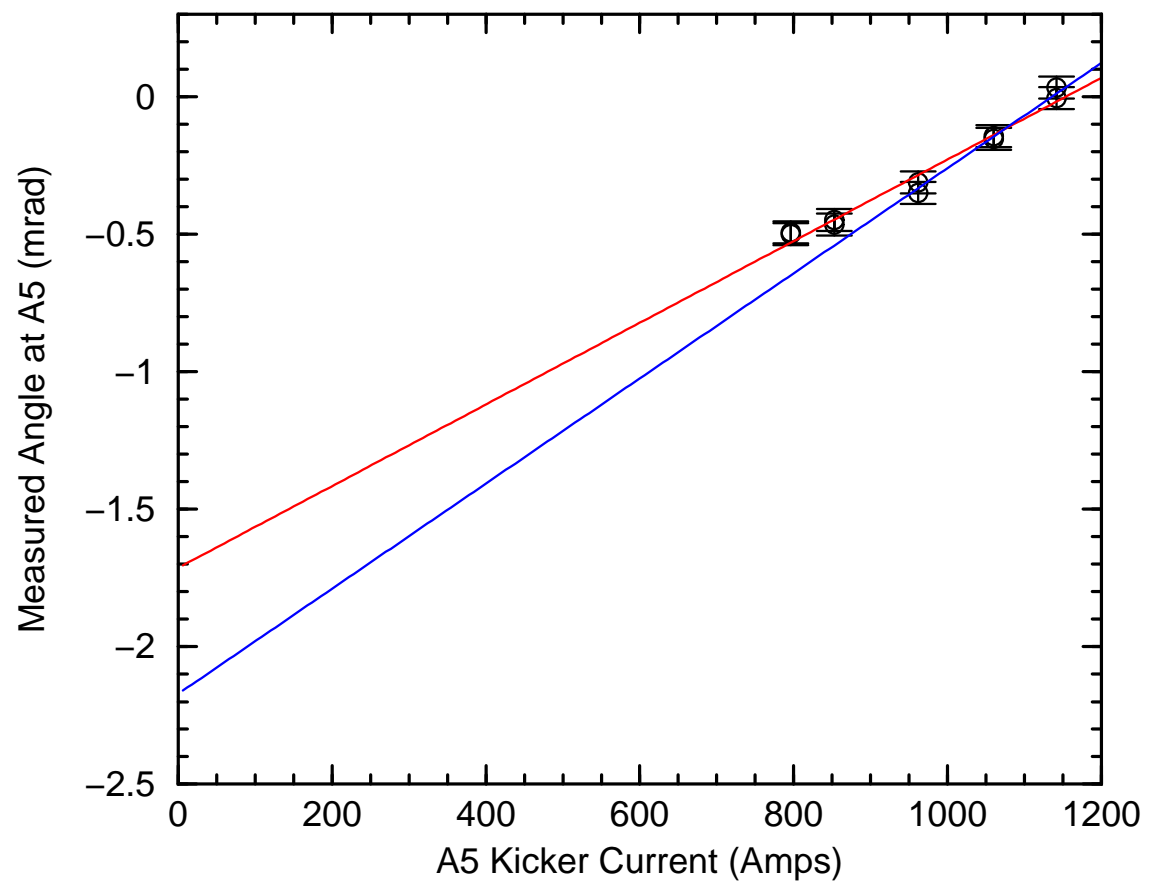

Figure 11: Data Set 3 with Fitted Lines

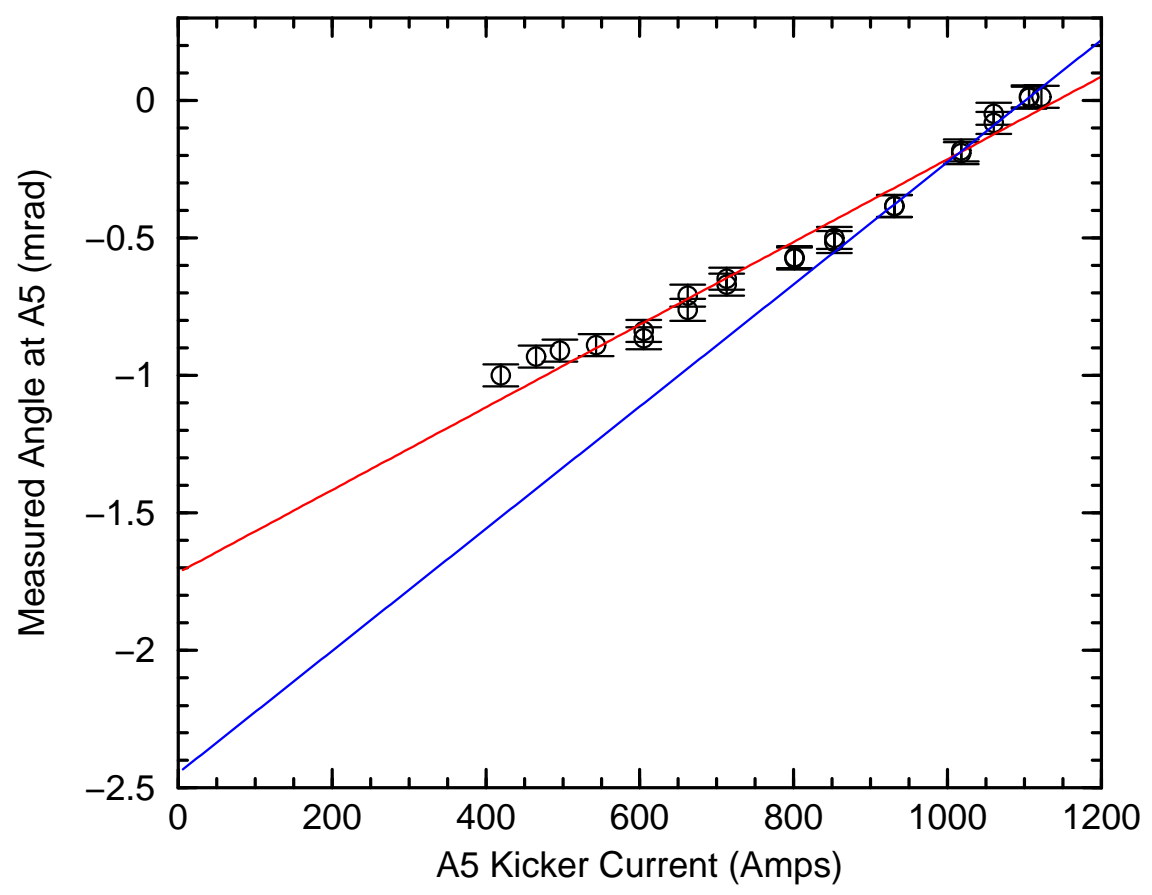

Figure 12: Data Set 4 with Fitted Lines 